\title{
Directed Proof Generation for Machine Code ${ }^{\star}$
}

\author{
Aditya Thakur ${ }^{1}$, Junghee Lim $^{1}$, Akash Lal ${ }^{2}$, Amanda Burton ${ }^{1}$, \\ Evan Driscoll ${ }^{1}$, Matt Elder ${ }^{1, \star \star}$, Tycho Andersen ${ }^{1}$, and Thomas Reps ${ }^{1,3, \star \star \star}$ \\ 1 University of Wisconsin; Madison, WI, USA \\ 2 Microsoft Research India; Bangalore, India \\ 3 GrammaTech, Inc.; Ithaca, NY, USA
}

\begin{abstract}
We present the algorithms used in McVeto (Machine-Code VErification TOol), a tool to check whether a stripped machinecode program satisfies a safety property. The verification problem that MCVETO addresses is challenging because it cannot assume that it has access to (i) certain structures commonly relied on by source-code verification tools, such as control-flow graphs and call-graphs, and (ii) meta-data, such as information about variables, types, and aliasing. It cannot even rely on out-of-scope local variables and return addresses being protected from the program's actions. What distinguishes MCVETO from other work on software model checking is that it shows how verification of machine-code can be performed, while avoiding conventional techniques that would be unsound if applied at the machine-code level.
\end{abstract}

\section{Introduction}

Recent research has led to new kinds of tools for analyzing programs for bugs and security vulnerabilities. In these tools, program analysis conservatively answers the question "Can the program reach a bad state?" Many impressive results have been achieved; however, the vast majority of existing tools analyze source code, whereas most programs are delivered as machine code. If analysts wish to vet such programs for bugs and security vulnerabilities, tools for analyzing machine code are needed.

Machine-code analysis presents many new challenges. For instance, at the machine-code level, memory is one large byte-addressable array, and an analyzer must handle computed - and possibly non-aligned - addresses. It is crucial to track array accesses and updates accurately; however, the task is complicated by the fact that arithmetic and dereferencing operations are both pervasive and inextricably intermingled. For instance, if local variable $\mathrm{x}$ is at offset -12 from the activation record's frame pointer (register ebp), an access on $\mathrm{x}$ would be turned

* Supported, in part, by NSF under grants CCF-\{0540955, 0810053, 0904371\}, by ONR under grants N00014-\{09-1-0510, 09-1-0776\}, by ARL under grant W911NF09-1-0413, and by AFRL under grant FA9550-09-1-0279.

** Supported by an NSF Graduate Fellowship.

${ }^{\star \star \star}$ T. Reps has an ownership interest in GrammaTech, Inc., which has licensed elements of the technology reported in this publication. 
into an operand [ebp-12]. Evaluating the operand first involves pointer arithmetic ("ebp-12") and then dereferencing the computed address ("[.]"). On the other hand, machine-code analysis also offers new opportunities, in particular, the opportunity to track low-level, platform-specific details, such as memorylayout effects. Programmers are typically unaware of such details; however, they are often the source of exploitable security vulnerabilities.

The algorithms used in software model checkers that work on source code [5 156] would be be unsound if applied to machine code. For instance, before starting the verification process proper, SLAM 5] and BLAST 15 perform flowinsensitive (and possibly field-sensitive) points-to analysis. However, such analyses often make unsound assumptions, such as assuming that the result of an arithmetic operation on a pointer always remains inside the pointer's original target. Such an approach assumes - without checking - that the program is ANSI $\mathrm{C}$ compliant, and hence causes the model checker to ignore behaviors that are allowed by some compilers (e.g., arithmetic is performed on pointers that are subsequently used for indirect function calls; pointers move off the ends of structs or arrays, and are subsequently dereferenced). A program can use such features for good reasons - e.g., as a way for a C program to simulate subclassing - but they can also be a source of bugs and security vulnerabilities.

This paper presents the techniques that we have implemented in a model checker for machine code, called MCVETO (Machine-Code VErification TOol). MCVETO uses directed proof generation (DPG) 13 to find either an input that causes a (bad) target state to be reached, or a proof that the bad state cannot be reached. (The third possibility is that MCVETO fails to terminate.)

What distinguishes the work on MCVETO is that it addresses a large number of issues that have been ignored in previous work on software model checking, and would cause previous techniques to be unsound if applied to machine code. The contributions of our work can be summarized as follows:

1. We show how to verify safety properties of machine code while avoiding a host of assumptions that are unsound in general, and that would be inappropriate in the machine-code context, such as reliance on symbol-table, debugging, or type information, and preprocessing steps for (a) building a precomputed, fixed, interprocedural control-flow graph (ICFG), or (b) performing points-to/alias analysis.

2. MCVETO builds its (sound) abstraction of the program's state space onthe-fly, performing disassembly one instruction at a time during state-space exploration, without static knowledge of the split between code vs. data. (It does not have to be prepared to disassemble collections of nested branches, loops, procedures, or the whole program all at once, which is what can confuse conventional disassemblers [20].)

The initial abstraction has only two abstract states, defined by the predicates "PC = target" and "PC $\neq$ target" (where "PC" denotes the program counter). The abstraction is gradually refined as more of the program is 
exercised (\$3). MCVETO can analyze programs with instruction aliasing 1 because it builds its abstraction of the program's state space entirely on-the-fly (3.1). Moreover, MCVETO is capable of verifying (or detecting flaws in) selfmodifying code (SMC). With SMC there is no fixed association between an address and the instruction at that address, but this is handled automatically by MCVETO's mechanisms for abstraction refinement. To the best of our knowledge, MCVETO is the first model checker to handle SMC.

3. MCVETO introduces trace generalization, a new technique for eliminating families of infeasible traces (\$3.1). Compared to prior techniques that also have this ability [14, our technique involves no calls on an SMT solver, and avoids the potentially expensive step of automaton complementation.

4. MCVETO introduces a new approach to performing DPG on multi-procedure programs (3.3). Godefroid et al. 12 presented a declarative framework that codifies the mechanisms used for DPG in SYNERGY [13], DASH [6], and SMASH 12] (which are all instances of the framework). In their framework, interprocedural DPG is performed by invoking intraprocedural DPG as a subroutine. In contrast, MCVETO's algorithm lies outside of that framework: the interprocedural component of MCVETO uses (and refines) an infinite graph, which is finitely represented and queried by symbolic operations.

5. We developed a language-independent algorithm to identify the aliasing condition relevant to a property in a given state (3.4). Unlike previous techniques [6], it applies when static names for variables/objects are unavailable.

Items 1 and 2 address execution details that are typically ignored (unsoundly) by source-code analyzers. Items 3, 4, and 5] are applicable to both source-code and machine-code analysis. MCVETO is not restricted to an impoverished language. In particular, it handles pointers and bit-vector arithmetic.

Organization. 92 contains a brief review of DPG. 33 describes the new DPG techniques used in MCVETO. \$4 describes how different instances of MCVETO are generated automatically from a specification of the semantics of an instruction set. $\$ 5$ presents experimental results. $\$ 6$ discusses related work. $\$ 7$ concludes.

\section{Background on Directed Proof Generation (DPG)}

Given a program $P$ and a particular control location target in $P$, DPG returns either an input for which execution leads to target or a proof that target is unreachable (or DPG does not terminate). Two approximations of $P$ 's state space are maintained:

- A set $T$ of concrete traces, obtained by running $P$ with specific inputs. $T$ underapproximates $P$ 's state space.

- A graph $G$, called the abstract graph, obtained from $P$ via abstraction (and abstraction refinement). $G$ overapproximates $P$ 's state space.

${ }^{1}$ Programs written in instruction sets with varying-length instructions, such as $\mathrm{x} 86$, can have "hidden" instructions starting at positions that are out of registration with the instruction boundaries of a given reading of an instruction stream [20]. 
Nodes in $G$ are labeled with formulas; edges are labeled with program statements or program conditions. One node is the start node (where execution begins); another node is the target node (the goal to reach). Information to relate the under- and overapproximations is also maintained: a concrete state $\sigma$ in a trace in $T$ is called a witness for a node $n$ in $G$ if $\sigma$ satisfies the formula that labels $n$.

If $G$ has no path from start to target, then DPG has proved that target is unreachable, and $G$ serves as the proof. Otherwise, DPG locates a frontier: a triple $(n, I, m)$, where $(n, m)$ is an edge on a path from start to target such that $n$ has a witness $w$ but $m$ does not, and $I$ is the instruction on $(n, m)$. DPG either performs concrete execution (attempting to reach target) or refines $G$ by splitting nodes and removing certain edges (which may prove that target is unreachable). Which action to perform is determined using the basic step from directed test generation 10, which uses symbolic execution to try to find

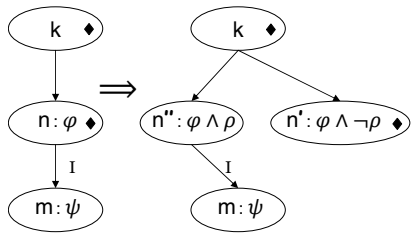

Fig. 1. The general refinement step across frontier $(n, I, m)$. The presence of a witness is indicated by a " $\gg$ " inside of a node. an input that allows execution to cross frontier $(n, I, m)$. Symbolic execution is performed over symbolic states, which have two components: a path constraint, which represents a constraint on the input state, and a symbolic map, which represents the current state in terms of input-state quantities. DPG performs symbolic execution along the path taken during the concrete execution that produced witness $w$ for $n$; it then symbolically executes $I$, and conjoins to the path constraint the formula obtained by evaluating $m$ 's predicate $\psi$ with respect to the symbolic map. It calls an SMT solver to determine if the path constraint obtained in this way is satisfiable. If so, the result is a satisfying assignment that is used to add a new execution trace to $T$. If not, DPG refines $G$ by splitting node $n$ into $n^{\prime}$ and $n^{\prime \prime}$, as shown in Fig. 1.

Refinement changes $G$ to represent some non-connectivity information: in particular, $n^{\prime}$ is not connected to $m$ in the refined graph (see Fig. 1). Let $\psi$ be the formula that labels $m, c$ be the concrete witness of $n$, and $S_{n}$ be the symbolic state obtained from the symbolic execution up to $n$. DPG chooses a formula $\rho$, called the refinement predicate, and splits node $n$ into $n^{\prime}$ and $n^{\prime \prime}$ to distinguish the cases when $n$ is reached with a concrete state that satisfies $\rho\left(n^{\prime \prime}\right)$ and when it is reached with a state that satisfies $\neg \rho\left(n^{\prime}\right)$. The predicate $\rho$ is chosen such that (i) no state that satisfies $\neg \rho$ can lead to a state that satisfies $\psi$ after the execution of $I$, and (ii) the symbolic state $S_{n}$ satisfies $\neg \rho$. Condition (i) ensures that the edge from $n^{\prime}$ to $m$ can be removed. Condition (ii) prohibits extending the current path along $I$ (forcing the DPG search to explore different paths). It also ensures that $c$ is a witness for $n^{\prime}$ and not for $n^{\prime \prime}$ (because $c$ satisfies $S_{n}$ ) - and thus the frontier during the next iteration must be different. 


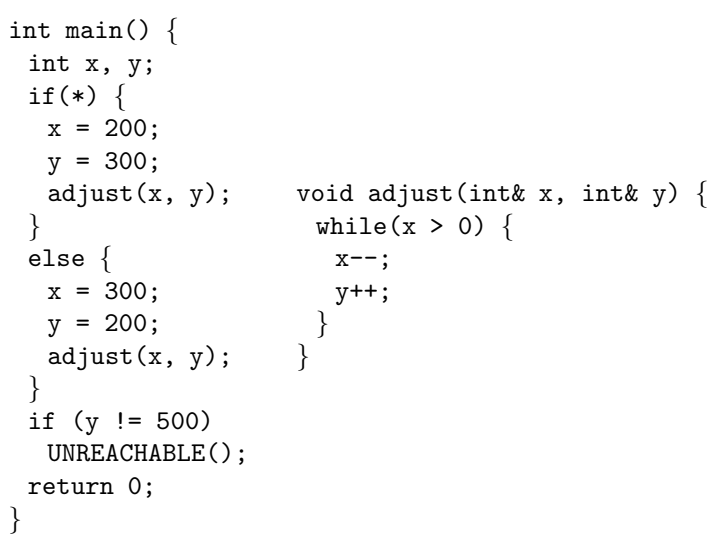

(a)

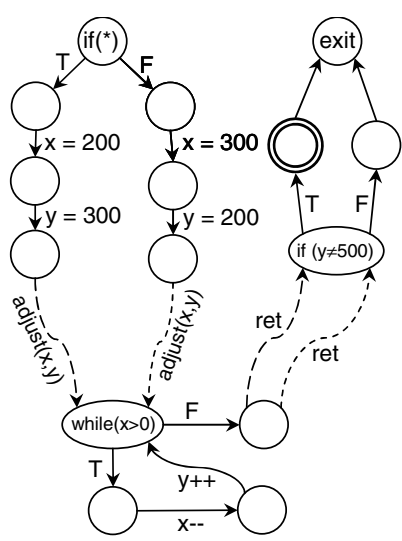

(b)

Fig. 2. (a) A program with a non-deterministic branch; (b) the program's ICFG

\section{McVeto}

This section explains the methods used to achieve contributions 15 listed in \$1. While MCVETO was designed to provide sound DPG for machine code, a number of its novel features are also useful for source-code DPG. Thus, to make the paper more accessible, our running example is the $\mathrm{C}++$ program in Fig. 2 . It makes a non-deterministic choice between two blocks that each call procedure adjust, which loops - decrementing $\mathrm{x}$ and incrementing $\mathrm{y}$. Note that the affine relation $x+y=500$ holds at the two calls on adjust, the loop-head in adjust, and the branch on $\mathrm{y} !=500$.

Representing the Abstract Graph. The infinite abstract graph used in MCVETO is finitely represented as a nested word automaton (NWA) [2] and queried by symbolic operations. As discussed in 3.1 the key property of NWAs for abstraction refinement is that, even though they represent matched call/return structure, they are closed under intersection 2]. That is, given NWAs $A_{1}$ and $A_{2}$, one can construct an NWA $A_{3}$ such that $L\left(A_{3}\right)=L\left(A_{1}\right) \cap L\left(A_{2}\right)$.

In our NWAs, the alphabet consists of all possible machine-code instructions. In addition, we annotate each state with a predicate. Operations on NWAs extend cleanly to accommodate the semantics of predicates - e.g., the $\cap$ operation labels a product state $\left\langle q_{1}, q_{2}\right\rangle$ with the conjunction of the predicates on states $q_{1}$ and $q_{2}$. In MCVETO's abstract graph, we treat the value of the PC as data; consequently, predicates can refer to the value of the PC (see Fig. 33).

\subsection{Abstraction Refinement via Trace Generalization}

In a source-code model checker, the initial overapproximation of a program's state space is often the program's ICFG. Unfortunately, for machine code it is difficult to create an accurate ICFG a priori because of the use of indirect 


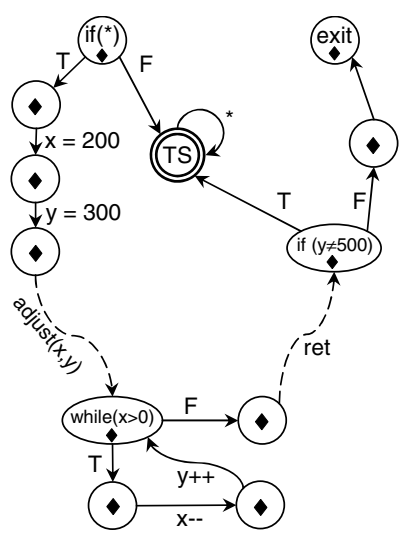

(a)

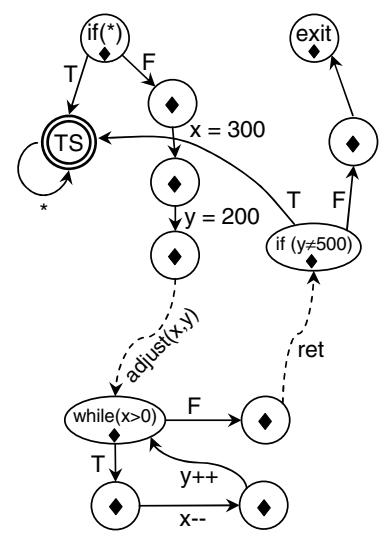

(b)

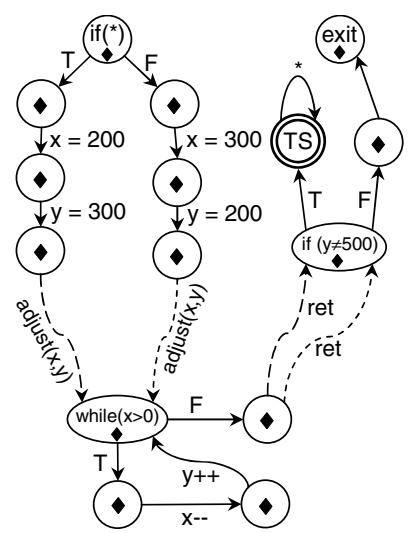

(c)

Fig. 4. (a) and (b) Two generalized traces, each of which reaches the end of the program. (c) The intersection of the two generalized traces. (A "४" indicates that a node has a witness.)

jumps, jump tables, and indirect function calls - as well as more esoteric features, such as instruction aliasing and SMC. For this reason, MCVETO begins with the degenerate NWA-based abstract graph $G_{0}$ shown in Fig. 3] which overapproximates the program's state space; i.e., $G_{O}$ accepts an overapproximation of the set of minima 2 traces that reach target. The abstract graph is refined during the state-space exploration carried out by MCVETO.

To avoid having to disassemble collections of nested branches, loops, procedures, or the whole program all at once, MCVETO performs trace-based disassembly: as concrete traces are generated during DPG, instructions are disassembled one at a time by decoding the current bytes of memory starting at the value of the PC. Each indirect jump or indirect call encountered can be resolved to a specific address. Trace-based disassembly is one of the techniques that allows MCVETO to handle self-modifying code.

MCVETO uses each concrete trace $\pi \in$ $T$ to refine abstract graph $G$. As men-

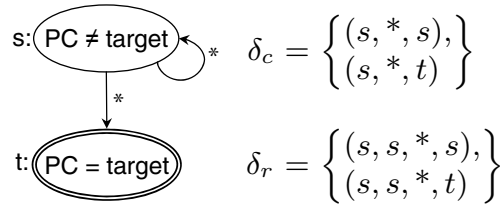

(a)

Fig. 3. (a) Internal-transitions in the initial NWA-based abstract graph $G_{0}$ created by McVETo; (b) call- and return-transitions in $G_{0}$. is a wild-card symbol that matches all instructions. tioned in 92 , the set $T$ of concrete traces underapproximates the program's state space, whereas $G$ represents an overapproximation of the state space. MCVETO repeatedly solves instances of the following trace-generalization problem:

\footnotetext{
${ }^{2}$ A trace $\tau$ that reaches target is minimal if $\tau$ does not have a proper prefix that reaches target.
} 
Given a trace $\pi$, which is an underapproximation of the program, convert $\pi$ into an NWA-based abstract graph $G_{\pi}$ that is an overapproximation of the program.

We create $G_{\pi}$ by "folding" $\pi$-grouping together all nodes with the same PC value, and augmenting it in a way that overapproximates the portion of the program not explored by $\pi$ (denoted by $\pi /[\mathrm{PC}]$ ); see Figs. 4 (a) and (b) and Fig. 5. In particular, $G_{\pi}$ contains one accepting state, called $T S$ (for "target surrogate"). TS is an accepting state because it represents target, as well as all non-target locations not visited by $\pi$. (Trace generalization for SMC is discussed in [25, §3.1].)

We now have two overapproximations, the original abstract graph $G$ and folded trace $G_{\pi}$. Thus, by performing $G:=G \cap G_{\pi}$, information about the portion of the program explored by $\pi$ is incorporated into $G$, producing a third, improved overapproximation; see Fig. 4(c). (Equivalently, intersection eliminates the family of infeasible traces represented by the complement of $G_{\pi}$; however, because we already have $G_{\pi}$ in hand, no automaton-complementation operation is required-cf. [14.)

The issue of how one forms an NWPrefix from an instruction sequence-i.e., identifying the nesting structure - is handled by a policy in the trace-recovery tool for classifying each position as an internal-, call-, or return-position. Currently, for reasons discussed in \$3.5. we use the following policy: the position of any form of call instruction is a call-position; the position of any form of ret instruction is a return-position. In essence, MCVETO uses call and ret instructions to restrict the instruction sequences considered. If these match the program's actual instruction sequences, we obtain the benefits of the NWA-based approach - especially the reuse of information among refinements of a given procedure. The basic MCVETO algorithm is stated as Alg. 1,

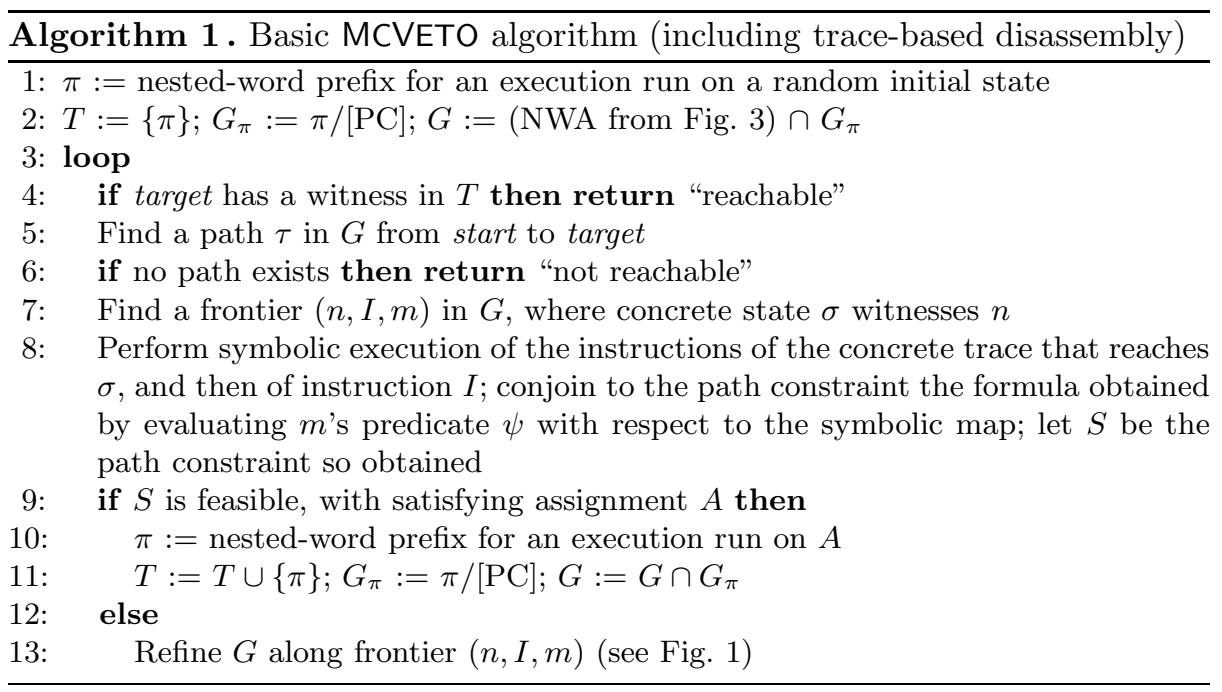


Definition 1. A trace $\pi$ that does not reach target is represented by (i) a nestedword prefix $(w, \rightsquigarrow)$ over instructions ([25, App. A]), together with (ii) an array of $P C$ values, $P C[1 . .|w|+1]$, where $P C[|w|+1]$ has the special value HALT if the trace terminated execution. Internal-steps, call-steps, and return-steps are triples of the form $\langle P C[i], w[i], P C[i+1]\rangle, 1 \leq i<|w|$, depending on whether $i$ is an internalposition, call-position, or return-position, respectively. Given $\pi$, we construct $G_{\pi} \stackrel{\text { def }}{=}$ $\pi /[P C]$ as follows:

1. All positions $1 \leq k<|w|+1$ for which $P C[k]$ has a given address a are collapsed to a single NWA state $q_{a}$. All such states are rejecting states (the target was not reached).

2. For each internal-step $\langle a, I, b\rangle, G_{\pi}$ has an internal-transition $\left(q_{a}, I, q_{b}\right)$.

3. For each call-step $\left\langle a_{c}\right.$, call, $\left.a_{e}\right\rangle, G_{\pi}$ has a call-transition $\left(q_{a_{c}}\right.$, call,$\left.q_{a_{e}}\right)$. ("call" stands for whatever instruction instance was used in the call-step.)

4. For each return-step $\left\langle a_{x}\right.$, ret, $\left.a_{r}\right\rangle$ for which the PC at the call predecessor holds address $a_{c}, G_{\pi}$ has a return-transition $\left(q_{a_{x}}, q_{a_{c}}\right.$, ret,$\left.q_{a_{r}}\right)$. ("ret" stands for whatever instruction instance was used in the return-step.)

5. $G_{\pi}$ contains one accepting state, called TS (for "target surrogate"). TS is an accepting state because it represents target, as well as all the non-target locations that $\pi$ did not explore.

6. $G_{\pi}$ contains three "self-loops": $\left(T S,{ }^{*}, T S\right) \in \delta_{i},(T S, *, T S) \in \delta_{c}$, and $\left(T S, T S,{ }^{*}, T S\right) \in \delta_{r}$. (We use "*" in the latter two transitions because there are many forms of call and ret instructions.)

7. For each unmatched instance of a call-step $\left\langle a_{c}\right.$, call, $\left.a_{e}\right\rangle, G_{\pi}$ has a returntransition $\left(T S, q_{a_{c}},{ }^{*}, T S\right)$. (We use * because any kind of ret instruction could appear in the matching return-step.)

8. Let $B_{b}$ denote a (direct or indirect) branch that takes branch-direction $b$.

- If $\pi$ has an internal-step $\left\langle a, B_{b}, c\right\rangle$ but not an internal-step $\left\langle a, B_{\neg b}, d\right\rangle, G_{\pi}$ has an internal-transition $\left(q_{a}, B_{\neg b}, T S\right)$.

- For each internal-step $\left\langle a, B_{T}, c\right\rangle$, where $B$ is an indirect branch, $G_{\pi}$ has an internal-transition $\left(q_{a}, B_{T}, T S\right)$.

9. For each call-step $\left\langle a_{c}\right.$, call, $\left.a_{e}\right\rangle$ where call is an indirect call, $G_{\pi}$ has a calltransition $\left(q_{a_{c}}\right.$, call, $\left.T S\right)$.

10. If $P C[|w|+1] \neq H A L T, G_{\pi}$ has an internal-transition $\left(q_{P C[|w|]}, I, T S\right)$, where "I" stands for whatever instruction instance was used in step $|w|$ of $\pi$. (We assume that an uncompleted trace never stops just before a call or ret.)

11. If $P C[|w|+1]=H A L T, G_{\pi}$ has an internal-transition $\left(q_{P C[|w|]}, I\right.$, Exit $)$, where "I" stands for whatever instruction instance was used in step $|w|$ of $\pi$ and Exit is a distinguished non-accepting state.

Fig. 5. Definition of the trace-folding operation $\pi /[\mathrm{PC}]$

\subsection{Speculative Trace Refinement}

Motivated by the observation that DPG is able to avoid exhaustive loop unrolling if it discovers the right loop invariant, we developed mechanisms to discover candidate invariants from a folded trace, which are then incorporated into the abstract graph via NWA intersection. Although they are only candidate invariants, they are introduced into the abstract graph in the hope that they are 


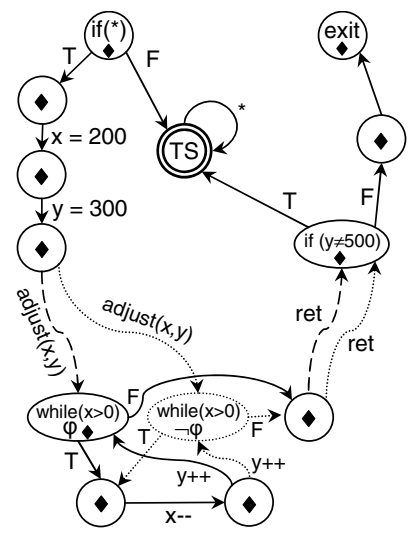

(a)

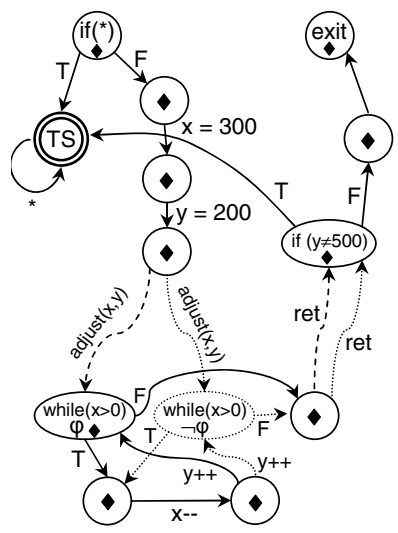

(b)

Fig. 6. Fig. 4(a) and (b) with the loop-head in adjust split with respect to the candidate invariant $\varphi \stackrel{\text { def }}{=} x+y=500$

invariants for the full program. The basic idea is to apply dataflow analysis to a graph obtained from the folded trace $G_{\pi}$. The recovery of invariants from $G_{\pi}$ is similar in spirit to the computation of invariants from traces in Daikon [9], but in MCVETO they are computed ex post facto by dataflow analysis on the folded trace. While any kind of dataflow analysis could be used in this fashion, MCVETO currently uses two analyses:

- Affine-relation analysis [21] is used to obtain linear equalities over registers and a set of memory locations, $V$. $V$ is computed by running aggregate structure identification [22] on $G_{\pi}$ to obtain a set of inferred memory variables $M$, then selecting $V \subseteq M$ as the most frequently accessed locations in $\pi$.

- An analysis based on strided-interval arithmetic 23 is used to discover range and congruence constraints on the values of individual registers and memory locations.

The candidate invariants are used to create predicates for the nodes of $G_{\pi}$. Because an analysis may not account for the full effects of indirect memory references on the inferred variables, to incorporate a discovered candidate invariant $\varphi$ for node $n$ into $G_{\pi}$ safely, we split $n$ on $\varphi$ and $\neg \varphi$. Again we have two overapproximations: $G_{\pi}$, from the folded trace, augmented with the candidate invariants, and the original abstract graph $G$. To incorporate the candidate invariants into $G$, we perform $G:=G \cap G_{\pi}$; the $\cap$ operation labels a product state $\left\langle q_{1}, q_{2}\right\rangle$ with the conjunction of the predicates on states $q_{1}$ of $G$ and $q_{2}$ of $G_{\pi}$.

Fig. 6 shows how the candidate affine relation $\varphi \stackrel{\text { def }}{=} x+y=500$ would be introduced at the loop-head of adjust in the generalized traces from Figs. 4(a) and (b). (Relation $\varphi$ does, in fact, hold for the portions of the state space explored by Figs. 4(a) and (b).) With this enhancement, subsequent steps of DPG will be able to show that the dotted loop-heads (labeled with $\neg \varphi$ ) can never be reached 


\begin{tabular}{|c|c|c|c|}
\hline $\begin{array}{l}\text { int } \mathrm{y} ; \\
\text { void } \operatorname{baz}()\{ \\
\qquad \begin{array}{l}\mathrm{y}=0 ; \\
\mathrm{y}^{++} ;\end{array} \\
\mathrm{y}^{--} ;\end{array}$ & $\begin{array}{l}\text { Void lotsaBaz(int a)\{ } \\
\text { y=0; } \\
\text { if }(a>0) \operatorname{baz}() ; \\
\text { if }(a>1) \operatorname{baz}() ; \\
\text { if }(a>2) \operatorname{baz}() ; \\
\text { if }(a>3) \operatorname{baz}() ; \\
\text { if }(y !=0) \\
\text { ERR: return; } \\
\text { \} }\end{array}$ & $\begin{array}{l}\text { int } \operatorname{bar} 1()\{ \\
\quad \text { int } i, r=0 ; \\
\quad \text { for }(i=0 ; i<100 ; i++)\{ \\
\quad \operatorname{complicated}() ; r++; \\
\text { return } r ; \\
\text { \} } \\
\text { int } \operatorname{bar} 2()\{\text { return } 10 ;\}\end{array}$ & $\begin{array}{l}\text { void foo(int } \mathrm{x})\{ \\
\text { int } \mathrm{y} ; \\
\text { if }(\mathrm{x}==0) \mathrm{y}=\operatorname{bar} 2() \text {; } \\
\text { else } \mathrm{y}=\operatorname{bar} 1() ; \\
\text { if }(\mathrm{y}==10) \\
\text { ERR: return; } \\
\text { \} }\end{array}$ \\
\hline
\end{tabular}

(a)

(b)

Fig. 7. Programs that illustrate the benefit of using a conceptually infinite abstract graph

from start. In addition, the predicate $\varphi$ on the solid loop-heads enables DPG to avoid exhaustive loop unrolling to show that the true branch of $y !=500$ can never be taken.

\subsection{Symbolic Methods for Interprocedural DPG}

In other DPG systems 13612, interprocedural DPG is performed by invoking intraprocedural DPG as a subroutine. In contrast, MCVETO analyzes a representation of the entire program (refined on-the-fly), which allows it to reuse all information from previous refinement steps. For instance, in the program shown in Fig. [7(a), procedure lotsaBaz makes several calls to baz. By invoking analysis once for each call site on baz, a tool such as DASH has to re-learn that y is set to 0 . In contrast, MCVETO only needs to learn this once and gets automatic reuse at all call sites. Note that such reuse is achieved in a different way in SMASH [12, which makes use of explicit procedure summaries. However, because the split between local and global variables is not known when analyzing machine code, it is not clear to us how MCVETO could generate such explicit summaries.

Furthermore, SMASH is still restricted to invoking intraprocedural analysis as a subroutine, whereas MCVETO is not limited to considering frontiers in just a single procedure: at each stage, it is free to choose a frontier in any procedure. To see why such freedom can be important, consider the example in Fig. 7(b) (where target is ERR). DASH might proceed as follows. The initial test uses $[\mathrm{x} \mapsto 42]$, which goes through bar1, but does not reach target. After a few iterations, the frontier is the call to bar1, at which point DASH is invoked on bar1 to prove that the return value is not 10 . The subproof takes a long time because of the complicated loop in bar1. In essence, DASH gets stuck in bar1 without recourse to an easier way to reach target. MCVETO can make the same choices, and would start to prove the same property for the return value of bar1. However, refinements inside of bar 1 cause the abstract graph to grow, and at some point, if the policy is to pick a frontier closest to target, the frontier switches to one in main that is closer to target - in particular, the true branch of the if-condition $\mathrm{x}==0$. MCVETO will be able to extend that frontier by running a test with $[\mathrm{x} \mapsto$ $0]$, which will go through bar2 and reach target. The challenge that we face to support such flexibility is how to select the frontier while accounting for 
paths that reflect the nesting structure of calls and returns. As discussed in [25] $\S 3.3]$, by doing computations via automata, transducers, and pushdown systems, MCVETO can find the set of all frontiers, as well as identify the $k$ closest frontiers.

\subsection{A Language-Independent Approach to Aliasing Relevant to a Property}

This section describes how MCVETO identifies - in a language-independent way suitable for use with machine code - the aliasing condition relevant to a property in a given state. There are two challenges to defining an appropriate notion of aliasing condition for use with machine code: (i) int-valued and address-valued quantities are indistinguishable at runtime, and (ii) arithmetic on addresses is used extensively.

Suppose that the frontier is $(n, I, m), \psi$ is the formula on $m$, and $S_{n}$ is the symbolic state obtained via symbolic execution of a concrete trace that reaches $n$. For source code, Beckman et al. 6 identify aliasing condition $\alpha$ by looking at the relationship, in $S_{n}$, between the addresses written to by $I$ and the ones used in $\psi$. However, their algorithm for computing $\alpha$ is language-dependent: their algorithm has the semantics of $\mathrm{C}$ implicitly encoded in its search for "the addresses written to by I". In contrast, as explained below, we developed an alternative, languageindependent approach, both to identifying $\alpha$ and computing $\operatorname{Pre}_{\alpha}$.

To simplify the discussion, suppose that a concrete machine-code state is represented using two maps $M: I N T \rightarrow I N T$ and $R: R E G \rightarrow I N T$. Map $M$ represents memory, and map $R$ represents the values of machine registers. We use the standard theory of arrays to describe (functional) updates and accesses on maps, e.g., update $(m, k, d)$ denotes the map $m$ with index $k$ updated with the value $d$, and $\operatorname{access}(m, k)$ is the value stored at index $k$ in $m$. (We use the notation $m(r)$ as a shorthand for access $(m, r)$.) We also use the standard axiom from the theory of arrays: $\left(\operatorname{update}\left(m, k_{1}, d\right)\right)\left(k_{2}\right)=$ ite $\left(k_{1}=k_{2}, d, m\left(k_{2}\right)\right)$, where ite is an if-then-else term. Suppose that $I$ is "mov [eax] , 5" (which corresponds to *eax $=5$ in source-code notation) and that $\psi$ is $(M(R(\mathrm{ebp})-8)+M(R(\mathrm{ebp})-12)=10) 3$ First, we symbolically execute $I$ starting from the identity symbolic state $S_{i d}=[M \mapsto M, R \mapsto R]$ to obtain the symbolic state $S^{\prime}=[M \mapsto \operatorname{update}(M, R(\operatorname{eax}), 5), R \mapsto R]$. Next, we evaluate $\psi$ under $S^{\prime}$-i.e., perform the substitution $\psi\left[M \leftarrow S^{\prime}(M), R \leftarrow S^{\prime}(R)\right]$. For instance, the term $M(R(\mathrm{ebp})-8)$, which denotes the contents of memory at address $R(\mathrm{ebp})-8$, evaluates to (update $(M, R(\mathrm{eax}), 5))(R(\mathrm{ebp})-8)$. From the axiom for arrays, this simplifies to ite $(R(\mathrm{eax})=R(\mathrm{ebp})-8,5, M(R(\mathrm{ebp})-8))$. Thus, the evaluation of $\psi$ under $S^{\prime}$ yields

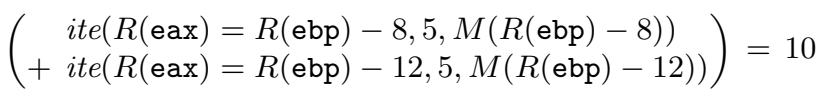

This formula equals $\operatorname{Pre}(I, \psi)[18$.

${ }^{3}$ In $\mathrm{x} 86$, ebp is the frame pointer, so if program variable $\mathrm{x}$ is at offset -8 and $\mathrm{y}$ is at offset $-12, \psi$ corresponds to $\mathrm{x}+\mathrm{y}=10$. 
The process described above illustrates a general property: for any instruction $I$ and formula $\psi$, Pre $(I, \psi)=\psi\left[M \leftarrow S^{\prime}(M), R \leftarrow S^{\prime}(R)\right]$, where $S^{\prime}=\mathrm{SE} \llbracket I \rrbracket S_{i d}$ and SE $\llbracket \cdot \rrbracket$ denotes symbolic execution [18].

The next steps are to identify $\alpha$ and to create a simplified formula $\psi^{\prime}$ that weakens $\operatorname{Pre}(I, \psi)$. These are carried out simultaneously during a traversal of $\operatorname{Pre}(I, \psi)$ that makes use of the symbolic state $S_{n}$ at node $n$. We illustrate this on the example discussed above for a case in which $S_{n}(R)=$ [eax $\mapsto R($ ebp $\left.)-8\right]$ (i.e., continuing the scenario from footnote 3. eax holds \&x). Because the iteterms in Eqn. (1) were generated from array accesses, ite-conditions represent possible constituents of aliasing conditions. We initialize $\alpha$ to true and traverse Eqn. (1). For each subterm $t$ of the form ite $\left(\varphi, t_{1}, t_{2}\right)$ where $\varphi$ definitely holds in symbolic state $S_{n}, t$ is simplified to $t_{1}$ and $\varphi$ is conjoined to $\alpha$. If $\varphi$ can never hold in $S_{n}, t$ is simplified to $t_{2}$ and $\neg \varphi$ is conjoined to $\alpha$. If $\varphi$ can sometimes hold and sometimes fail to hold in $S_{n}, t$ and $\alpha$ are left unchanged.

In our example, $R($ eax $)$ equals $R(\mathrm{ebp})-8$ in symbolic state $S_{n}$; hence, applying the process described above to Eqn. (11) yields

$$
\begin{aligned}
\psi^{\prime} & =(5+M(R(\text { ebp })-12)=10) \\
\alpha & =(R(\text { eax })=R(\text { ebp })-8) \wedge(R(\text { eax }) \neq R(\text { ebp })-12)
\end{aligned}
$$

The formula $\alpha \Rightarrow \psi^{\prime}$ is the desired refinement predicate $\operatorname{Pre}_{\alpha}(I, \psi)$.

In practice, we found it beneficial to use an alternative approach, which is to perform the same process of evaluating conditions of ite terms in $\operatorname{Pre}(I, \psi)$, but to use one of the concrete witness states $W_{n}$ of frontier node $n$ in place of symbolic state $S_{n}$. The latter method is less expensive (it uses formula-evaluation steps in place of SMT solver calls), but generates an aliasing condition specific to $W_{n}$ rather than one that covers all concrete states described by $S_{n}$.

Both approaches are language-independent because they isolate where the instruction-set semantics comes into play in $\operatorname{Pre}(I, \psi)$ to the computation of $S^{\prime}=$ $\mathrm{SE} \llbracket I \rrbracket S_{i d}$; all remaining steps involve only purely logical primitives. Although our algorithm computes $\operatorname{Pre}(I, \psi)$ explicitly, that step alone does not cause an explosion in formula size; explosion is due to repeated application of Pre. In our approach, the formula obtained via $\operatorname{Pre}(I, \psi)$ is immediately simplified to create first $\psi^{\prime}$, and then $\alpha \Rightarrow \psi^{\prime}$.

Byte-Addressable Memory. When memory is byte-addressable, the actual memory-map type is INT32 $\rightarrow$ INT8. This complicates matters because accessing (updating) a 32-bit quantity in memory translates into four contiguous 8-bit accesses (updates). [25, §3.4] shows how a result equivalent to Eqn. (2) is obtained for a memory-map of type INT32 $\rightarrow$ INT8.

\subsection{Soundness Guarantee}

The soundness argument for MCVETO is more subtle than it otherwise might appear because of examples like the one shown in Fig. 8. The statement ${ }^{*}(\& r+2)=r$; overwrites foo's return address, and MakeChoice returns a random 32-bit number. At the end of foo, half the runs return normally to main. For the other half, 
the ret instruction at the end of foo serves to call bar. The problem is that for a run that returns normally to main after trace generalization and intersection with $G_{0}$, there is no frontier. Consequently, half of the runs of MCVETO, on average, would erroneously report that location ERR is unreachable.

MCVETO uses the following policy $P$ for classifying execution steps: (a) the position of any form of call instruction is a callposition; (b) the position of any form of ret instruction is a return-position. Our goals are (i) to define a property $Q$ that is compatible with $P$ in the sense that MCVETO can check for violations of $Q$ while checking only $N W$ Prefix paths ([25, App. A]), and (ii) to establish a soundness guarantee: either MCVETO reports that $Q$ is violated (along with an input that demonstrates it), or it reports that target is reachable (again with an input that demonstrates it), or it correctly reports that $Q$ is invariant and target is unreachable. To define $Q$, we augment the instruction-set semantics with an auxiliary stack. Initially, the auxiliary stack is empty; at each call, a copy

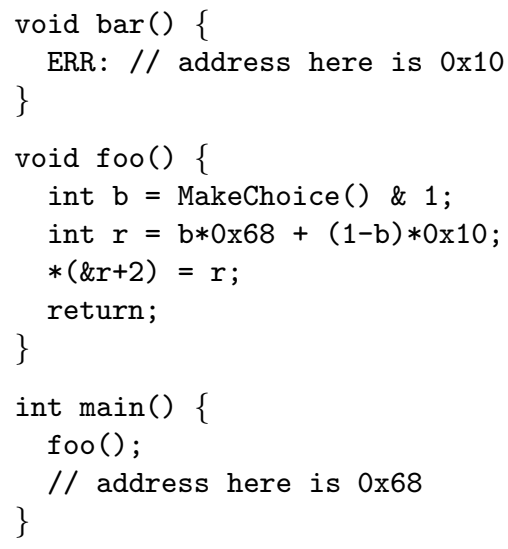

Fig. 8. ERR is reachable, but only along a path in which a ret instruction serves to perform a call of the return address pushed on the processor stack is also pushed on the auxiliary stack; at each ret, the auxiliary stack is popped.

Definition 2. An acceptable execution (AE) under the instrumented semantics is one in which at each ret instruction (i) the auxiliary stack is non-empty, and (ii) the address popped from the processor stack matches the address popped from the auxiliary stack.

In the instrumented semantics, a flag $\mathrm{V}$ is set whenever the program performs an execution step that violates either condition (i) or (ii) of Defn. 2, Instead of the initial NWA shown in Fig. 3, we use a similar two-state NWA that has states $q_{1}: \mathrm{PC} \neq$ target $\wedge \neg \mathrm{V}$ and $q_{2}: \mathrm{PC}=$ target $\vee \mathrm{V}$, where $q_{1}$ is non-accepting and $q_{2}$ is accepting. In addition, we add one more rule to the trace-generalization construction for $G_{\pi}$ from Fig. [5]

12. For each return-step $\left\langle a_{x}\right.$, ret,$\left.a_{r}\right\rangle, G_{\pi}$ has an internal-transition $\left(q_{a_{x}}\right.$, ret, $\left.T S\right)$. As shown below, these modifications cause the DPG algorithm to also search for traces that are AE violations.

\section{Theorem 1 (Soundness of MCVETO)}

1. If MCVETO reports "AE violation" (together with an input $S$ ), execution of $S$ performs an execution that is not an $A E$.

2. If MCVETO reports "bug found" (together with an input $S$ ), execution of $S$ performs an $A E$ to target.

3. If MCVETO reports "OK", then (a) the program performs only AEs, and (b) target cannot be reached during any $A E$. 
Sketch of Proof: If a program has a concrete execution trace that is not AE, there must exist a shortest non-AE prefix, which has the form "NWPrefix ret" where either (i) the auxiliary stack is empty, or (ii) the return address used by ret from the processor stack fails to match the return address on the auxiliary stack. At each stage, the abstract graph used by MCVETO accepts an overapproximation of the program's shortest non-AE execution-trace prefixes. This is true of the initial graph $G_{0}$ because internal transitions have wild-card symbols. Moreover, each folded trace $G_{\pi}=\pi /[\mathrm{PC}]$ accepts traces of the form "NWPrefix ret" due to the addition of internal transitions to $T S$ for each ret instruction (item 12 above). NWA intersection of two sound overapproximations leads to a refined sound overapproximation. Therefore, when MCVETO has shown that no accepting state is reachable, it has also proved that the program has no $\mathrm{AE}$ violations.

\section{Implementation}

The MCVETO implementation incorporates all of the techniques described in $₫ 3$. The implementation uses only language-independent techniques; consequently, MCVETO can be easily retargeted to different languages. The main components of MCVETO are language-independent in two different dimensions:

1. The MCVETO DPG driver is structured so that one only needs to provide implementations of primitives for concrete and symbolic execution of a language's constructs, plus a handful of other primitives (e.g., Pre $\alpha$ ). Consequently, this component can be used for both source-level languages and machine-code languages.

2. For machine-code languages, we used two tools that generate the required implementations of the primitives for concrete and symbolic execution from descriptions of the syntax and concrete operational semantics of an instruction set. The abstract syntax and concrete semantics are specified using TSL (Transformer Specification Language) [19]. Translation of binary-encoded instructions to abstract syntax trees is specified using a tool called ISAL (Instruction Set Architecture Language).

In addition, we developed language-independent solutions to each of the issues in MCVETO, such as identifying the aliasing condition relevant to a specific property in a given state (\$3.4). Consequently, our implementation acts as a Yacc-like tool for creating versions of MCVETO for different languages: given a description of language $L$, a version of MCVETO for $L$ is generated automatically. We created two specific instantiations of MCVETO from descriptions of the Intel x86 and PowerPC instruction sets. To perform symbolic queries on the conceptuallyinfinite abstract graph $([25, \S 3.3])$, the implementation uses OpenFst [1] (for transducers) and WALi [16] (for WPDSs).

\section{Experiments}

Our experiments (see Fig. 9) were run on a single core of a single-processor quad-core 3.0 GHz Xeon computer running Windows XP, configured so that a 


\begin{tabular}{|c|c|c|c|c|c|c|}
\hline \multicolumn{2}{|l|}{ Program } & \multicolumn{5}{|c|}{ 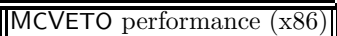 } \\
\hline Name & Outcome & \#Instrs & $\mathrm{CE}$ & $\mathrm{SE}$ & Ref & time \\
\hline blast2/blast2 & timeout & 98 & ** & *** & & \\
\hline $\mathrm{CH}-0$ & & 49 & $* *$ & $* *$ & $* *$ & * \\
\hline EACH-1 & counterex. & 49 & 1 & 0 & 0 & 0.125 \\
\hline slam1/slam1 & proof & 84 & 15 & 129 & 307 & 203 \\
\hline smc1/smc1-REACH-0* & & 21 & 1 & 60 & 188 & 959 \\
\hline smc1/smc1-REACH-1* & counterex. & 21 & 1 & 0 & 0 & 0.016 \\
\hline ex5/ex & counterex. & 48 & 2 & 10 & 38 & 3.05 \\
\hline doubleloopdep/count-COUNT-6 & counterex. & 31 & 7 & 11 & 13 & 11.5 \\
\hline ep/count-COUNT-7 & countes & 31 & 7 & 11 & 13 & 11.6 \\
\hline UNT-8 & counte & 31 & 7 & 11 & 13 & 11.6 \\
\hline doubleloopdep/count-COUNT-9 & counterex. & 31 & 7 & 11 & 13 & 11.7 \\
\hline inter.synerg & time & 253 & $* *$ & $* *$ & $* *$ & *** \\
\hline inter.synergy/berkeley & counterex. & 104 & 5 & 13 & 16 & 3.95 \\
\hline inter.synergy/cars & proof & 196 & 11 & 118 & 349 & 188 \\
\hline inter.synergy/efm & timeout & 188 & $* *$ & $* *$ & $* *$ & $* *$ \\
\hline CASE-0 & & 50 & 3 & 24 & 75 & 8.5 \\
\hline cert/un & counterex. & 120 & 2 & 6 & 12 & 9.55 \\
\hline instraliasing/instraliasing-REACH-0 & proof & 46 & 2 & 36 & 126 & 15.0 \\
\hline g/instraliasing-REACH-1 & counter & 46 & 2 & 17 & 55 & 5.86 \\
\hline 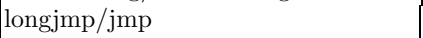 & AE viol. & 74 & 1 & 0 & 0 & 0.015 \\
\hline overview0/overview & proof & 49 & 2 & 31 & 91 & 54.9 \\
\hline ic_bench/ex5 & & 33 & 3 & 7 & 13 & 2.27 \\
\hline & & 30 & 1 & 55 & 146 & 153 \\
\hline small_static_bench/ex8 & proof & 89 & 4 & 17 & 46 & 6.31 \\
\hline simp_bad & counterex. & 1067 & 1 & 0 & 0 & 0.094 \\
\hline verise & proof & 1068 & $* *$ & $* *$ & $* *$ & \\
\hline clobber_ret_ac & AE viol. & 43 & 4 & 9 & 18 & 2.13 \\
\hline clobber_ret_ar & AE viol. & 35 & 2 & 2 & 5 & 0.625 \\
\hline clobber_ret_addr/clobber-CASE-9 & proof & 35 & 1 & 5 & 21 & 1.44 \\
\hline
\end{tabular}

Fig. 9. MCVeto experiments. The columns show whether MCVETo returned a proof, counterexample, or an AE violation (Outcome); the number of instructions (\#Instrs); the number of concrete executions (CE); the number of symbolic executions (SE), which also equals the number of calls to the YICES solver; the number of refinements (Ref), which also equals the number of Pre $_{\alpha}$ computations; and the total time (in seconds). ${ }^{*}$ SMC test case. ${ }^{* *}$ Exceeded twenty-minute time limit.

user process has $4 \mathrm{~GB}$ of memory. They were designed to test various aspects of a DPG algorithm and to handle various intricacies that arise in machine code (some of which are not visible in source code). We compiled the programs with Visual Studio 8.0, and ran MCVETO on the resulting object files (without using symbol-table information) 4

The examples ex5, ex6, and ex8 are from the NECLA Static Analysis Benchmarks. The examples barber, berkeley, cars, efm are multi-procedure versions of the larger examples on which SYNERGY [13] was tested. (SYNERGY was tested using single-procedure versions only.) Instraliasing illustrates the ability to handle instruction aliasing. (The instruction count for this example was obtained via static disassembly, and hence is only approximate.) Smc1 illustrates the ability of MCVETO to handle self-modifying code. Underflow is taken from a DHS tutorial on security vulnerabilities. It illustrates a strncpy vulnerability.

\footnotetext{
${ }^{4}$ The examples are available at www.cs.wisc.edu/wpis/examples/McVeto
} 
The examples are small, but challenging. They demonstrate MCVETO's ability to reason automatically about low-level details of machine code using a sequence of sound abstractions. The question of whether the cost of soundness is inherent, or whether there is some way that the well-behavedness of (most) code could be exploited to make the analysis scale better is left for future research.

\section{Related Work}

Machine-Code Analyzers Targeted at Finding Vulnerabilities. A substantial amount of work exists on techniques to detect security vulnerabilities by analyzing source code. Less work exists on vulnerability detection for machine code. Kruegel et al. [17] developed a system for automating mimicry attacks; it uses symbolic execution to discover attacks that can give up and regain execution control by modifying the contents of the data, heap, or stack so that the application is forced to return control to injected attack code at some point after the execution of a system call. Cova et al. 8] used that platform to detect security vulnerabilities in x86 executables via symbolic execution.

Prior work exists on directed test generation for machine code [117]. In contrast, MCVETO implements directed proof generation. Unlike directed-testgeneration tools, MCVETO is goal-directed, and works by trying to refute the claim "no path exists that connects program entry to a given goal state".

Machine-Code Model Checkers. SYNERGY applies to an x86 executable for a "single-procedure C program with only [int-valued] variables" [13] (i.e., no pointers). It uses debugging information to obtain information about variables and types, and uses Vulcan [24] to obtain a CFG. It uses integer arithmetic - not bit-vector arithmetic - in its solver. In contrast, MCVETO addresses the challenges of checking properties of stripped executables articulated in $\$ 1$

Our group developed two prior machine-code model checkers, CodeSurfer/x86 [4] and DDA/x86 [3]. Neither system uses underapproximation. For overapproximation, both use numeric static analysis and a different form of abstraction refinement.

Trace Generalization. The trace-generalization technique of $\$ 3.1$ has both similarities to and differences from the trace-refinement technique of Heizmann et al. 14]. Both techniques adopt a language-theoretic viewpoint and refine an overapproximation to eliminate families of infeasible concrete traces. However, trace generalization obtains the desired outcome in a substantially different way. For Heizmann et al., once a refutation automaton is constructed - which involves calling an SMT solver and an interpolant generator - refinement is performed by automaton complementation followed by automaton intersection. In contrast, our generalized traces are created by generalizing a feasible concrete trace to create directly a representation that overapproximates the set of minimal traces that reach target. Consequently, refinement by trace generalization involves no calls on an SMT solver, and avoids the potentially expensive step of automaton complementation. 


\section{Conclusion}

MCVETO resolves many issues that have been unsoundly ignored in previous work on software model checking. MCVETO addresses the challenge of establishing properties of the machine code that actually executes, and thus provides one approach to checking the effects of compilation and optimization on correctness. The contributions of the paper lie in the insights that went into defining the innovations in dynamic and symbolic analysis used in MCVETO: (i) sound disassembly and sound construction of an overapproximation (even in the presence of instruction aliasing and self-modifying code) (\$3.1), (ii) a new method to eliminate families of infeasible traces (\$3.1), (iii) a method to speculatively, but soundly, elaborate the abstraction in use (\$3.2), (iv) new symbolic methods to query the (conceptually infinite) abstract graph (\$3.3), and (v) a language-independent approach to $\operatorname{Pre}_{\alpha}$ (\$3.4). Not only are our techniques language-independent, the implementation is parameterized by specifications of an instruction set's semantics. By this means, MCVETO has been instantiated for both x86 and PowerPC.

\section{References}

1. Allauzen, C., Riley, M., Schalkwyk, J., Skut, W., Mohri, M.: OpenFst: A general and efficient weighted finite-state transducer library. In: Holub, J., Ždárek, J. (eds.) CIAA 2007. LNCS, vol. 4783, pp. 11-23. Springer, Heidelberg (2007)

2. Alur, R., Madhusudan, P.: Adding nesting structure to words. JACM 56 (2009)

3. Balakrishnan, G., Reps, T.: Analyzing stripped device-driver executables. In: Ramakrishnan, C.R., Rehof, J. (eds.) TACAS 2008. LNCS, vol. 4963, pp. 124-140. Springer, Heidelberg (2008)

4. Balakrishnan, G., Reps, T., Kidd, N., Lal, A., Lim, J., Melski, D., Gruian, R., Yong, S., Chen, C.-H., Teitelbaum, T.: Model checking x86 executables with CodeSurfer/x86 and WPDS++. In: Etessami, K., Rajamani, S.K. (eds.) CAV 2005. LNCS, vol. 3576, pp. 158-163. Springer, Heidelberg (2005)

5. Ball, T., Rajamani, S.: The SLAM toolkit. In: Berry, G., Comon, H., Finkel, A. (eds.) CAV 2001. LNCS, vol. 2102, p. 260. Springer, Heidelberg (2001)

6. Beckman, N., Nori, A., Rajamani, S., Simmons, R.: Proofs from tests. In: ISSTA (2008)

7. Brumley, D., Hartwig, C., Liang, Z., Newsome, J., Poosankam, P., Song, D., Yin, H.: Automatically identifying trigger-based behavior in malware. In: Botnet Detection. Springer, Heidelberg (2008)

8. Cova, M., Felmetsger, V., Banks, G., Vigna, G.: Static detection of vulnerabilities in x86 executables. In: Jesshope, C., Egan, C. (eds.) ACSAC 2006. LNCS, vol. 4186. Springer, Heidelberg (2006)

9. Ernst, M., Perkins, J., Guo, P., McCamant, S., Pacheco, C., Tschantz, M., Xiao, C.: The Daikon system for dynamic detection of likely invariants. SCP 69 (2007)

10. Godefroid, P., Klarlund, N., Sen, K.: DART: Directed automated random testing. In: PLDI (2005)

11. Godefroid, P., Levin, M., Molnar, D.: Automated whitebox fuzz testing. In: NDSS (2008) 
12. Godefroid, P., Nori, A., Rajamani, S., Tetali, S.: Compositional may-must program analysis: Unleashing the power of alternation. In: POPL (2010)

13. Gulavani, B., Henzinger, T., Kannan, Y., Nori, A., Rajamani, S.: SYNERGY: A new algorithm for property checking. In: Robshaw, M.J.B. (ed.) FSE 2006. LNCS, vol. 4047, pp. 117-127. Springer, Heidelberg (2006)

14. Heizmann, M., Hoenicke, J., Podelski, A.: Nested interpolants. In: POPL (2010)

15. Henzinger, T., Jhala, R., Majumdar, R., Sutre, G.: Lazy abstraction. In: POPL (2002)

16. Kidd, N., Lal, A., Reps, T.: WALi: The Weighted Automaton Library (2007), http://www.cs.wisc.edu/wpis/wpds/download.php

17. Kruegel, C., Kirda, E., Mutz, D., Robertson, W., Vigna, G.: Automating mimicry attacks using static binary analysis. In: USENIX Sec. Symp. (2005)

18. Lim, J., Lal, A., Reps, T.: Symbolic analysis via semantic reinterpretation. In: SPIN Workshop (2009)

19. Lim, J., Reps, T.: A system for generating static analyzers for machine instructions. In: Hendren, L. (ed.) CC 2008. LNCS, vol. 4959, pp. 36-52. Springer, Heidelberg (2008)

20. Linn, C., Debray, S.: Obfuscation of executable code to improve resistance to static disassembly. In: CCS (2003)

21. Müller-Olm, M., Seidl, H.: Analysis of modular arithmetic. In: Sagiv, M. (ed.) ESOP 2005. LNCS, vol. 3444, pp. 46-60. Springer, Heidelberg (2005)

22. Ramalingam, G., Field, J., Tip, F.: Aggregate structure identification and its application to program analysis. In: POPL (1999)

23. Reps, T., Balakrishnan, G., Lim, J.: Intermediate-representation recovery from low-level code. In: PEPM (2006)

24. Srivastava, A., Edwards, A., Vo, H.: Vulcan: Binary transformation in a distributed environment. MSR-TR-2001-50, Microsoft Research (April 2001)

25. Thakur, A., Lim, J., Lal, A., Burton, A., Driscoll, E., Elder, M., Andersen, T., Reps, T.: Directed proof generation for machine code. TR 1669, UW-Madison (April 2010) 